\title{
Dirofilaria repens eyepiece: a case in Calabria and diagnostic indications concerning dirofilariosis
}

\author{
Carlo Mancuso \\ Laboratorio Analisi, Ospedale Civile di Cetraro (CS)
}

Key Words: Dirofilaria repens, Nematode

Dirofilaria repens oculare: un caso in Calabria ed indicazioni diagnostiche relative alle dirofilariosi

\section{SUMMARY}

Dirofilaria repens is a nematode that affects dogs and other carnivores such as cats, wolves, foxes, which are final hosts. It is carried to the men by mosquitos (Anopheles, Culex and Aedes) and, particularly, by Aedes albopictus. It causes in the humans the formation of subcutaneous nodules and, at ocular conjunctiva level, the formation of cysts. Its diagnosis is made by methods of Molecular Biology because the identification based only on the morphology is very difficult. In the mounth of November 2012, comes to the first aid of Cetraro Hospital a old woman with a conjunctival cyst to right eye which causes pain, itch and lachrymation. From this cyst, is extracted a worm, identified as Dirofilaria repens. This diagnosis is confirmed by investigations of Molecular Biology discharged in the Superior Institute of Health of Rome.

\section{INTRODUZIONE}

Dirofilaria repens è un nematode tissutale ampiamente diffuso nel mondo, Italia compresa, specialmente in Piemonte e in Lombardia. Colpisce prevalentemente i cani, le volpi, i lupi ma anche i gatti localizzandosi a livello sottocutaneo.

E trasportato dall'animale all'uomo attraverso le zanzare del genere Anopheles, Culex, Aedes e, in particolare, attraverso l'Aedes albopictus meglio conosciuta come zanzara tigre. Nell'uomo si localizza a livello sottocutaneo e a livello sub congiuntivale, dove provoca la formazione di un nodulo o di una cisti sierosa da cui fuoriesce il verme adulto. L'identificazione della Dirofilaria basata solamente sulla morfologia è difficile: è necessario pertanto ricorrere ai metodi di Biologia Molecolare per identificare correttamente la specie. I geni nucleari e mitocondriali sono utili per l'identificazione di specie elmintiche e, in particolare, i geni mitocondriali sono spesso usati per l' $i$ dentificazione della Dirofilaria.

\section{Classificazione}

Le Dirofilarie appartengono essenzialmente a due specie, la Dirofilaria immitis e la Dirofilaria repens. Gli adulti di Dirofilaria immitis vivono nel cuore dell'ospite definitivo, di cui possono causare la morte quando sono in numero elevato. Gli adulti di Dirofilaria repens sono presenti nel tessuto sottocutaneo dell'ospite definitivo producendo microfilarie circolanti nel sangue che passano da un ospite all'altro (tra cui l'uomo) tramite le zanzare.

\section{Ciclo Vitale}

Il ciclo vitale della Dirofiliara repens si suddivide in cinque stadi che avvengono nell'ospite vertebrato e nella zanzara intesa sia come ospite intermedio, sia come vettore. Nel primo stadio, le femmine adulte, che si sono accoppiate, producono giornalmente 1000 larve che vengono immesse in circolo e che vengono assunte dalle zanzare con un pasto ematico. Nella zanzara, dopo 10-16 giorni, le larve passano allo stadio infettivo prima di essere reintrodotte nel nuovo ospite. Le filarie sono sottoposte ad ulteriori cambiamenti nell'insetto. Negli stadi finali di sviluppo, le larve sono inoculate di nuovo nell'ospite vertebrato durante un pasto ematico da parte dell'artropode.

Gli adulti di Dirofilaria repens risiedono nei tessuti sottocutanei dei cani e dei gatti prima di migrare al cuore, dove maturano in 6-7 mesi. Gli uomini sono ospiti accidentali perché i vermi adulti non possono raggiungere la maturità nel cuore o nella cute. La maggior parte delle larve introdotte negli uomini, sono candidate a morire.

\section{Diagnosi morfologica}

Il verme appare lungo da 4 a $12.5 \mathrm{~cm}$ e largo da 0.5-0.6 mm. Da un punto di vista macroscopico, ha un aspetto filiforme ed è di colore bianco.

Da un punto di vista microscopico, nella parte anteriore si individua bene la bocca e l'esofago. Caratteristiche della Dirofilaria repens sono le sporgenze longitudinali esterne simili a una ruota dentata, la cuticola spessa, e la muscolatura della parete esterna ben sviluppata. La sezione istologi-

\section{Corresponding author: Carlo Mancuso}

Laboratorio Analisi, Ospedale Civile, Cetraro (CS), Azienda Sanitaria Provinciale di Cosenza

Via Misasi, 40 - 87I00 Cosenza - Tel.: 098471223 - Cell.: 3382717962

E-mail: carlo.mancuso.58@alice.it 
ca del tessuto intorno al parassita mostra una marcata reazione infiammatoria con numerosi granulociti neutrofili ed eosinofili, linfociti, plasmacellule e macrofagi. Sono inoltre presenti numerose plasmacellule $\operatorname{IgE}$ positive nell'infiltrato e vicino all'interfaccia del parassita.

\section{Diagnosi con metodi sierologici}

La ricerca di anticorpi contro gli antigeni somatici è di fondamentale importanza per valutare l'efficacia della terapia. I test maggiormente adoperati sono quelli immunoenzimatici, basati sulla tecnica ELISA e quelli di immunomigrazione.

\section{Diagnosi con metodi di Biologia Molecolare}

Il DNA del parassita viene estratto adoperando un kit che si trova in commercio come ad es. il Qiagen genomic DNA extraction kit. Il DNA genomico viene diluito ad una concentrazione di $100 \mathrm{ng} /$ microlitro e 5 microlitri di questa soluzione viene ulteriormente diluito in 50 microlitri di volume di reazione di PCR (Polymerase Chain Reaction). La PCR amplifica un frammento di subunità di Citocromo C ossidasi 1 (cox1) usando una coppia di primers denominati UCO1F1 e UCO1R2. I prodotti della PCR possono essere confrontati con i dati della Gen Data Base Bank. In uno studio effettuato dal Dipartimento di Parassitologia Medica della Facoltà di Medicina e Chirurgia dell'Università di Hanoi, una porzione di Cox 1 della Dirofilaria repens vietnamita, comprendente 461 nucleotidi e 153 amminoacidi era comparata con la stessa porzione di Cox 1 della Dirofilaria repens italiana. Si otteneva una similitudine nella sequenza nucleotidica del $95 \%$.

\section{DESCRIZIONE DEL CASO CLINICO}

Nel Mese di Novembre 2012, giunge al Pronto Soccorso dell'Ospedale Civile di Cetraro (ASP di Cosenza) un'anziana signora che lamenta dolore, lacrimazione e fotofobia all'occhio destro. Mandata in consulenza nel reparto di Oculistica, si nota la presenza di una cisti localizzata a livello della congiuntiva da cui viene estratto un verme vitale, che viene inviato in Laboratorio Analisi per essere identificato. Dal punto di vista macroscopico, il parassita si presenta filiforme, di $8-10 \mathrm{~cm}$ di lunghezza, di colore bianco (figura I e II). Da un punto di vista microscopico, si notano le tipiche sporgenze longitudinali simili ad una ruota dentata e la muscolatura della parete ben sviluppata (figura III e IV). Tramite la Direzione Sanitaria Ospedaliera, il parassita viene spedito presso l'Istituto Superiore di Sanità in Roma dove vengono espletate le indagini di Biologia Molecolare che confermano la diagnosi di Dirofilaria repens. La signora, dopo un breve periodo di osservazio- ne, viene dimessa senza alcuna terapia in quanto la rimozione chirurgica del parassita, brillantemente eseguita dai nostri Oculisti, ha di fatto risolto ogni problema.

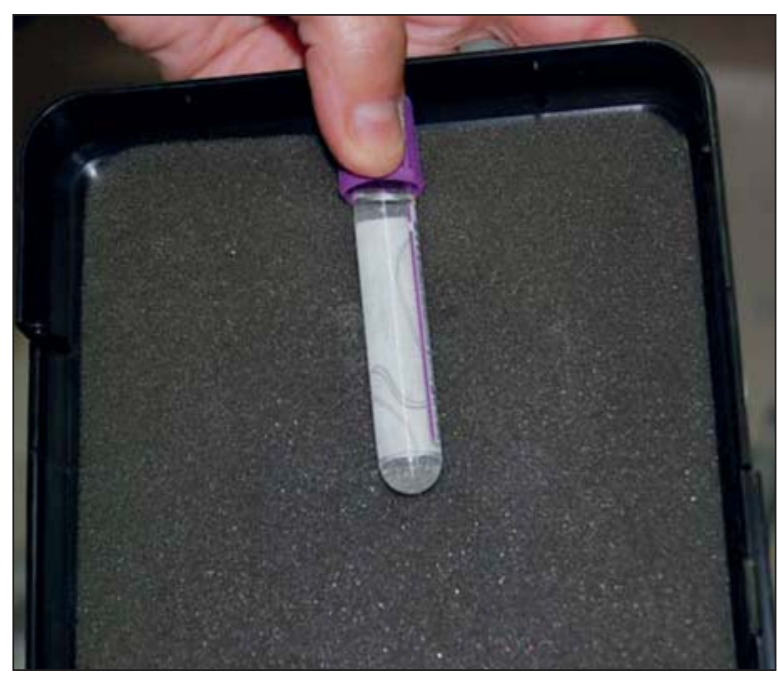

Figura I

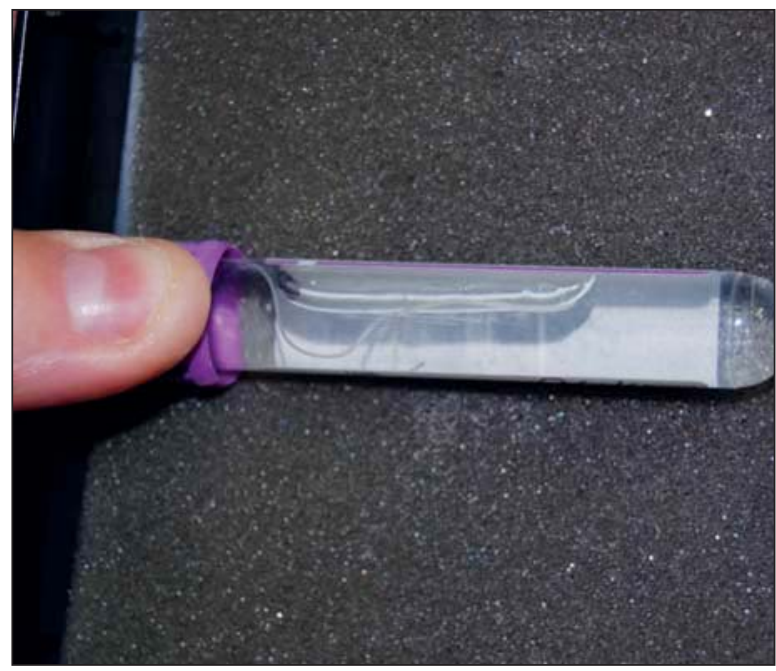

Figura II

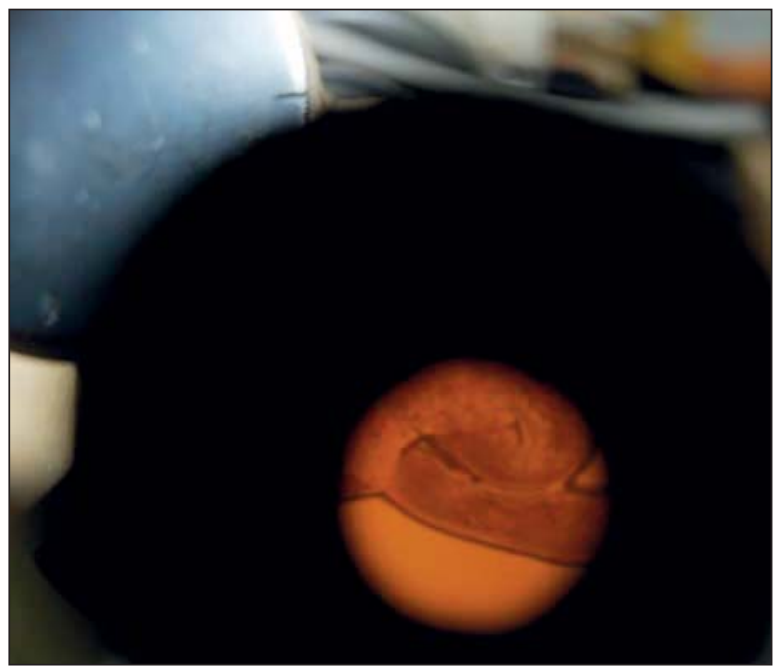

Figura II 


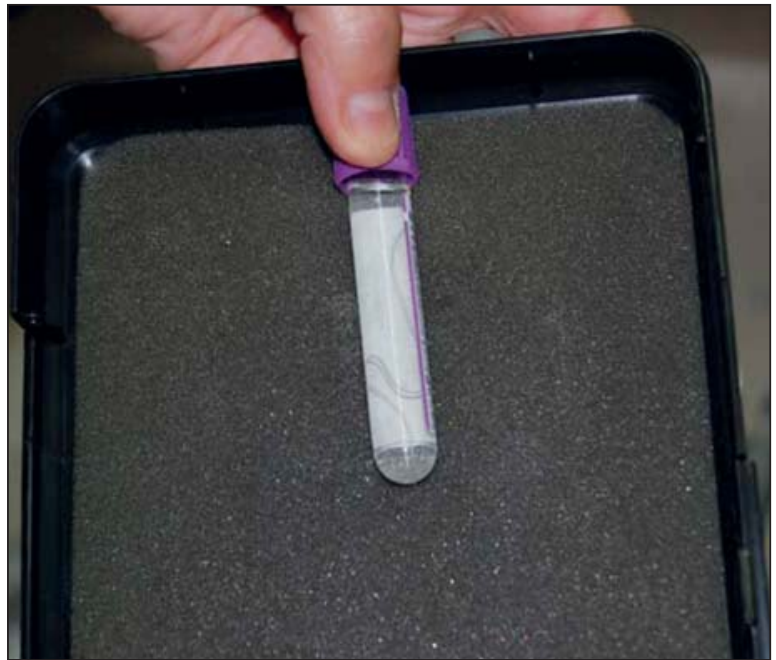

Figura IV

\section{BIBLIOGRAFIA}

1. Bernieri F, Crotti D, Galli D, Raglio A. Manuale illustrato di Diagnodtica Parassitologica. 2001; 4-5.

2. Cancrini G. Parassitologia medica illustrata. Lombardo Editore. 1996; 287-8.

3. Chandrasekharan NV, Karunanayake EH, Franzen L, Abeyewickreme W, Pettersson U. Dirofilaria repens: cloning and characterization of a repeated DNA sequence for the diagnosis of dirofilariasis in dogs. Exp Parasitology, 1994 May; 279-86.

4. Fodor E, Fok E, Maka E, Lukats O, Toth J. Recently recognized cases of ophthalmofilariasis in Hungary. Eur Jourof Ophthal, 2009; 19 (4): 675-8.

5. Kramer LH, Kartashev VV, Grandi G, et al. Human Subcutaneous Dirofilariasis, Russia. Center for Disease Control and Prevention (end/article/13/01/2006-0920).

6. Nugyen Van De, Thanh Hoa Le, Jong-Yil Chai. Dirofilaria repens in Vietnam. Detection of eye and subcutaneous tissue infection cases identified by morphology and molecular methods. Korean $J$ of Parasito. 2012; 50 (2): 137-41.

7. Otranto D, Brianti E, Gaglio G, Dantas-Torres F, Azzaro S, Giannetto S. Human ocular infection with Dirofilaria repens (Railliet and Henry 1911) in an Area Endemic for Canine Dirofilariasis. Am J Trop HyG, 2011 June 1; 84 (6): 1002-4.

8. Pampiglione S, Rivasi F, Angeli G, Boldorini R, Incensati RM, Pastomerlo R. Dirofilariosis due to Dirofilaria repens in Italy, an emergent zoonosis: report of 60new cases. Histopathology 2001; 38 (4): 344-54.

9. Popescu I, Tudose I, Racz P, Muntau B, Giurdaneanu C, Poppert S. Human Dirofilaria repens Infection in Romania: a case report. Case Reports in Infectious Disease Volume 2012 article ID 472976.

10. Poppert S, Hodapp M, Krueger A, et al. Dirofilaria repens infection and concomitant Meningoencephalitis. Center for Disease Control and Prevention (end/article/ 15/11/2009-093b). 